\title{
Idiopathic Thrombocytopenic Purpura \& Total Knee Arthroplasty: How Low Can You Go
}

Harkeerat Singh ${ }^{1}$, Kunalan Ganthel ${ }^{2}$

${ }^{1}$ Department of Orthopaedics, Traumatology \& Rehabilitation, Kulliyyah of

Medicine, International Islamic University Malaysia

${ }^{2}$ Hospital Kuala Lumpur

Presenter: Harkeerat Singh

Idiopathic thrombocytopenic purpura or ITP is a an autoimmune disorder with a reduction in circulating platelets, it subjects patients to spontaneous torrential bleeding even with trivial trauma. In this case report we share our experience conducting a TKR in a patient with refractory ITP. A 64-year-old patient with ITP complicated with history of ICB in 2001 presented with bilateral knee pain. Her platelet counts were $60 \times 10^{9} / \mathrm{L}$ despite previous steroidal and immunoglobulin therapy. After discussion haematologist a manual platelet count under microscopy had $15-20 / \mathrm{hpf}$ large platelets with estimated counts to be $100 \times 10^{9} / \mathrm{L}$. She underwent a cemented right TKR under general anaesthesia. No femoral/sciatic block was performed nor was a tourniquet applied fearing an intramuscular haematoma/ecchymosis. Two grams of intravenous tranexemic acid was given prior to the surgery and four units of platelets were transfused intraoperatively. Despite no tourniquet, she clotted very well and blood loss was minimal. Thereafter, ITP is an immune disorder where patients form antibodies towards their own platelets resulting in increased platelet destruction and a decrease in platelet formation. Remaining circulating platelets are bigger than usual platelets, however, their function are not impaired. ITP guidelines by the British Journal Of Haematology dictate a minimal platelet count of $50 \times 10^{9} / \mathrm{L}$ for minor surgical procedures and $80 \times 10^{9} / \mathrm{L}$ for major surgical procedures. It is still safe to perform TKR in ITP patients. However a combined approach with haematology is advocated as manual platelet counts give a better representation of the actual number of circulating platelets compared to routine blood counts. 